\title{
Synthesis and rotation barriers in 2, 6-Di-(o-anisyl) anisole
}

\author{
Takuhei Yamamoto ${ }^{a}$, Pi-Yu Chen ${ }^{a}$, Guangxin Lin ${ }^{a}$, Anna Błoch-Mechkour ${ }^{b}$, \\ Neil E. Jacobsen ${ }^{a}$, Thomas Bally ${ }^{b_{*}}$ and Richard S. Glass ${ }^{a *}$
}

\begin{abstract}
Variable temperature ${ }^{1} \mathrm{H}$ NMR spectroscopic studies of 2, 6-di(o-anisyl) anisole show syn and anti atropisomers at low temperature. The barrier for interconverting these isomers by rotation about the aryl-aryl bond, found by fitting the experimental data, is $41.2 \mathrm{~kJ} / \mathrm{mol}$.
\end{abstract}

Keywords: conformational analysis; dynamic NMR; quantum chemical calculations

\section{INTRODUCTION}

We recently reported ${ }^{[1]}$ atropisomerism in certain $m$-terphenyl thio- seleno- and telluro- ethers, such as 1a-c. The barriers for interconversion of the two syn and the anti atropisomers, respectively, were determined by variable temperature NMR spectroscopic studies.
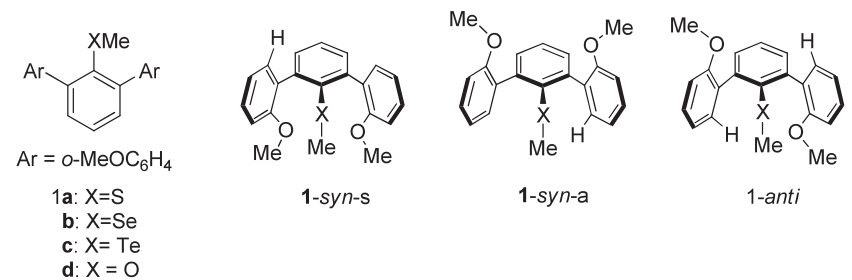

The barriers for this interconversion increase on going from $\mathrm{S}$ to Se to Te that is, $\mathbf{1 a} \rightarrow \mathbf{1} \mathbf{b} \rightarrow \mathbf{1}$ c. Calculations on $\mathbf{1 a}$ and $\mathbf{1} \mathbf{b}$ revealed that these barriers are due to rotation about the aryl-aryl bonds. Furthermore, the magnitude of this barrier is determined by the increase in the size of the chalcogen $(\mathrm{S}<\mathrm{Se}<\mathrm{Te})$ and not by that of the length of the aryl-chalcogen bond ( $\mathrm{S}-\mathrm{C}<\mathrm{Se}-\mathrm{C}<\mathrm{Te}-\mathrm{C}$ ). Notably absent from this study was the first-row chalcogen: oxygen. Since little work has been reported ${ }^{[2-5]}$ in these and related systems involving oxygen, we have synthesized and studied anisole derivative $\mathbf{1 d}$ and the results are reported in this note.

\section{RESULTS AND DISCUSSION}

Cram and coworkers ${ }^{[6]}$ first synthesized 1d, from bromodibenzofuran and Dyker and Kellner ${ }^{[7]}$ made it in modest yields in a mixture of products formed in an interesting Pd-catalyzed cascade reaction from 2-iodoanisole. We synthesized $\mathbf{1 d}$, using a Suzuki coupling reaction ${ }^{[8]}$ as shown below:
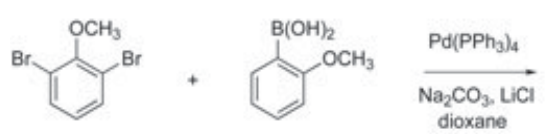

The ${ }^{1} \mathrm{H}$ and ${ }^{13} \mathrm{C}$ NMR spectra for 2,6-di-(o-anisyl)anisole were fully assigned on the basis of analysis of $1 D$ and 2D NMR spectra.
The 2D NMR spectra are found in the Supporting Information. The two singlets at $\delta 3.143$ and 3.816 were assigned to hydrogens of the methoxy groups on the central ring and the outer ring, respectively, based on their chemical shifts and integrations. The methoxy protons showed correlations in the heteronuclear single quantum coherence (HSQC) spectrum, which allowed assignment of the signals at $\delta 56.00$ and $\delta 60.75$ in the ${ }^{13} \mathrm{C}$ NMR spectrum to the outer ring and the center ring, respectively. The COSY correlations indicated the protons at $\delta 7.147$ and 7.231 are in the same ring and are assigned to $\mathrm{H} 4$ and $\mathrm{H} 3,5$ on the basis of the $A B_{2}$ splitting pattern and relative integrated areas. Crosspeaks in the HSQC spectrum of $\mathrm{H} 4$ with $\mathrm{C} 4$ and $\mathrm{H} 3,5$ with $\mathrm{C} 3,5$ allowed the assignment of the signal at $\delta 123.17$ and that at $\delta 131.66$ to $C 4$ and $\mathrm{C} 3,5$, respectively. The heteronuclear multiple bond correlation (HMBC) spectrum showed the methoxy protons on the center ring and on the outer ring correlates with quaternary carbons bound to each of the oxygens and hence assigned to $C 1$ ( $\delta$ 156.72) and $C 2^{\prime}\left(\delta\right.$ 157.45), respectively. The signal at $\delta 132.54$ in the ${ }^{13} \mathrm{C}$ NMR spectrum showed correlation with $\mathrm{H} 4$, but because there was no crosspeak in HSQC, this absorption was assigned to $\mathrm{C} 2,6$. In the ${ }^{1} \mathrm{H}$ NMR spectrum, on the basis of the chemical shifts due to the methoxy group and the observed splitting patterns, $\mathrm{H} 3$ ', $\mathrm{H}^{\prime}, \mathrm{H}^{\prime}$, and $\mathrm{H} 4^{\prime}$ correlations in the HSQC spectrum, could be assigned to the signals at $\delta 7.016,7.018,7.293$, and 7.357, respectively. On the basis of correlations of these signals in HSQC C3' $(\delta 111.34), C 5^{\prime}(\delta 120.79), C 6^{\prime}(\delta 131.85)$, and $C 4^{\prime}(\delta 129.23)$, signals could be assigned as shown. These assignments were further confirmed by crosspeaks in the ROESY spectrum of $\mathrm{H}^{\prime} / \mathrm{OCH} 3$ in the

\footnotetext{
* Correspondence to: Richard S. Glass, Department of Chemistry and Biochemistry, The University of Arizona, Tucson, AZ 85721, USA.

E-mail: rglass@email.arizona.edu

Thomas Bally, Department of Chemistry, University of Fribourg, CH-1700, Fribourg, Switzerland.

E-mail: thomas.bally@unifr.ch

a T. Yamamoto, P.-Y. Chen, G. Lin, N.E. Jacobsen, R.S. Glass Department of Chemistry and Biochemistry, The University of Arizona, Tucson AZ 85721, USA
}

b A. Błoch-Mechkour, T. Bally

Department of Chemistry, University of Fribourg, CH-1700, Fribourg, Switzerland 


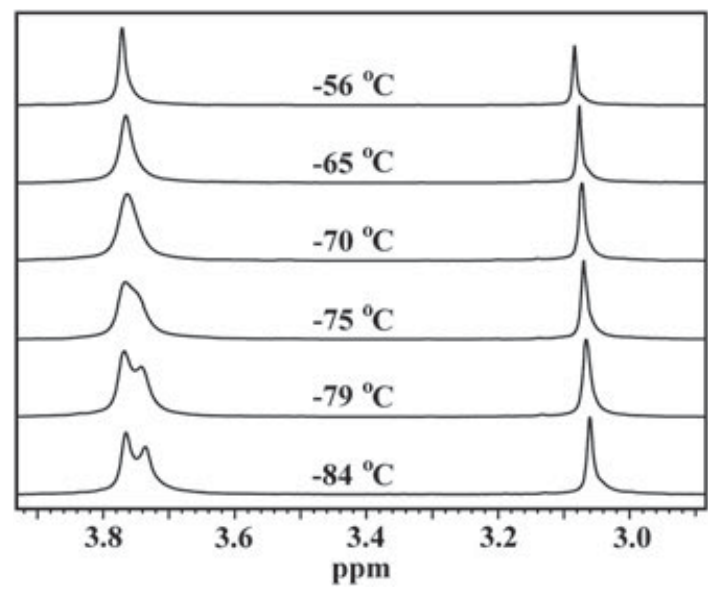

Figure 1. Variable temperature $300 \mathrm{MHz}^{1} \mathrm{H}$ NMR spectra of anisole $1 \mathbf{d}$ in $\mathrm{CD}_{2} \mathrm{Cl}_{2}$ (OMe region)

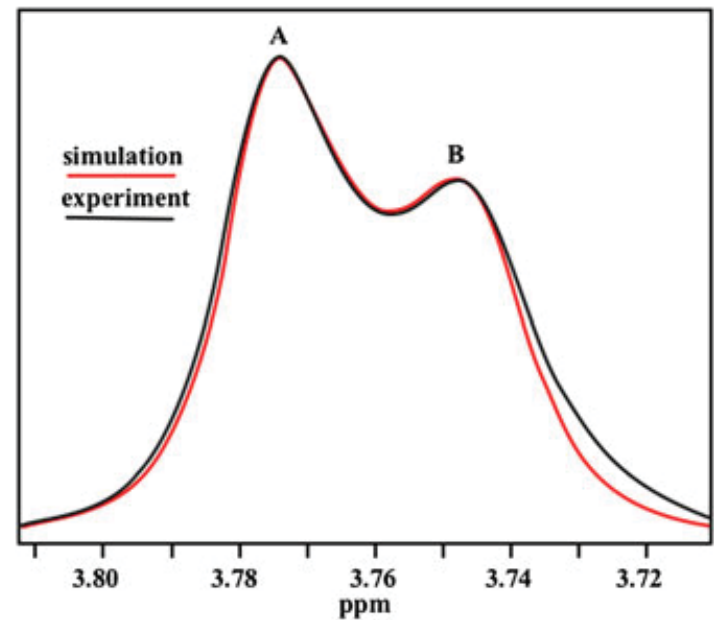

Figure 2. Experimental and simulated ${ }^{1} \mathrm{H}$ NMR OMe peaks of Ar moieties in $1 \mathbf{d}$ in $\mathrm{CH}_{2} \mathrm{Cl}_{2}$ at $-79^{\circ} \mathrm{C}(\mathrm{C}$, see text for parameters)

outer ring and correlations in the $\mathrm{HMBC}$ spectrum of $\mathrm{H}^{\prime}$ and $\mathrm{H} 5^{\prime} / \mathrm{C}^{\prime}$ and $\mathrm{H} 6^{\prime} / \mathrm{C} 2,6$. Finally, $\mathrm{H} 1^{\prime} / \mathrm{C} 1^{\prime}$ was assigned on the basis of no crosspeaks in the HSQC and correlation with $\mathrm{H} 3,5$ in the $\mathrm{HMBC}$ spectrum.

The variable temperature ${ }^{1} \mathrm{H}$ NMR spectra of $\mathbf{1} \mathbf{d}$ are shown in Figure 1. At room temperature the $o$-anisyl methoxy signals give rise to a singlet at $3.8 \mathrm{ppm}$ and their equivalence connotes rapid rotation about the aryl-aryl bond at this temperature. However, as the temperature is lowered the signal broadens and ultimately splits into two peaks. Analysis of the coalescence data provided an excellent fit of the observed and calculated spectra as shown in Figure 2. The fit around the critical coalescence temperature $\left(-79^{\circ} \mathrm{C}\right)$ gave the following fitting parameters and calculated barrier: line-width $1.5 \mathrm{~Hz}, v_{A}-v_{B} 11.2 \mathrm{~Hz}, \mathrm{k}_{A B}+k_{B A}$ $32.3 \mathrm{~s}^{-1}, \% \mathrm{~A}$ 54.7, $\Delta \mathrm{G}^{\neq}(\mathrm{A} \rightarrow \mathrm{B})=41.2 \mathrm{~kJ} / \mathrm{mol}$. As expected the barrier for $\mathbf{1 d}$ is substantially less than that reported for $\mathbf{1 a - c}$. As reported previously for these latter compounds ${ }^{[1]}$, a detailed analysis of the barrier was required for $\mathbf{1} \mathbf{d}$, because the variable temperature NMR spectra show that the chemical shifts vary significantly with temperature. Consequently, the WinDNMR program, ${ }^{[9]}$ which was used to obtain the parameters by fitting the $-79 \mathrm{C}$ data and, thus, to determine the activation barrier for interconversion of the atropisomers.
Interestingly, only one peak is observed for the methoxy group appended to the central ring in $\mathbf{1 d}$ at the lowest temperature recorded, whereas 1a-c show two signals for the SMe, SeMe and TeMe moieties, respectively, below their coalescence temperature. Apparently, the difference in chemical shifts for the OMe groups of the central ring for the syn and anti atropisomers is not resolved in our experiments. Note that, as before ${ }^{[1]}$ there are three possible atropisomers: one anti and two syn isomers ${ }^{[10]}$ but evidence is obtained for only two. This presumably results from rapid rotation about the aryl-O bond of the central ring.

\section{Molecular Modeling}

The structures of all three atropisomers and the transition states between them were optimized by the B3LYP/6-31G* and the B2PLYP-D/cc-pVDZ methods. The calculated structures are depicted in the Figure 3 (and in Table S2 of the Supporting Information, along with the three important dihedral angles). It is worth noting that there are two transition states for the automerization of the anti conformers which are attained when the central-OMe group rotates towards one or the other lateral-OMe group. However, the energy difference between these two transition states is less than $3 \mathrm{~kJ} / \mathrm{mol}$, and we will only consider the lower of the two, which appears in Figure 3. (In Figure S8 and Table S1 of the Supporting Information both transition states are shown). There is only one transition state between the two syn conformers because both of them have a plane of symmetry.

The relative enthalpies and free energies of the minima and transition states shown in Figure 3 are listed in Table 1. They were calculated both in the gas phase and in $\mathrm{CH}_{2} \mathrm{Cl}_{2}$ as a dielectric continuum solvent, but the results obtained from the two sets of calculations do not differ significantly. They show that the barriers for rotation around the central Ph-OMe bond, i.e. for the syn- $a \rightarrow$ syn-s interconversion and for the automerization of the anti conformer, are too low for these processes to be detected in the temperature range that is accessible in our NMR experiments, i.e. rapid equilibration between the syn- $a$ and syn-s conformers is to be expected.

We have previously reported ${ }^{[1]}$ that these barriers decrease on going from $X=S(\mathbf{1} \mathbf{a})$ to $X=S e(\mathbf{1} \mathbf{b})$ and we had attributed this to the increase of the $C-X$ bond length, which leads to a concomitant increase of the distance between the Me that is attached to chalcogen and the lateral phenyl group. By this logic the barrier for rotation of the lateral phenyl group should be the highest for $\mathrm{X}=\mathrm{O}$ (1d), but our present results show that this is not the case (the gas phase activation enthalpy for the the syn- $a \rightarrow$ syn-s interconversion, calculated by the same method, is $19.4 \mathrm{~kJ} / \mathrm{mol}$ for $\mathbf{1 a}$ and $16.5 \mathrm{~kJ} / \mathrm{mol}$ for $\mathbf{1 b}$. The corresponding numbers for the anti automerization are 19.0 and $15.3 \mathrm{~kJ} / \mathrm{mol}$, respectively).

We attempted to single out a reason for these peculiar trends by model calculations on the fragments from which compounds $\mathbf{1}$ are composed. These calculations showed that the steric repulsion between the Me group and the lateral phenyl group changes by less than $1 \mathrm{~kJ} / \mathrm{mol}$ along the series. The distortion of the central $\mathrm{Ph}-\mathrm{X}$-Me moiety in the transition state, relative to optimized structures, does show an increase on going from $X=O$ to $X=S$ and a decrease on going to $X=S e$, but this effect accounts only for about a third of the total change. Thus we conclude that the observed trends are due to a combination of causes which cannot be easily disentangled.

On the other hand, the calculated barriers for converting the syn- $a$ or syn-s to the anti conformers, i.e., for rotation of a lateral phenyl group, are much higher than the barriers for rotating 


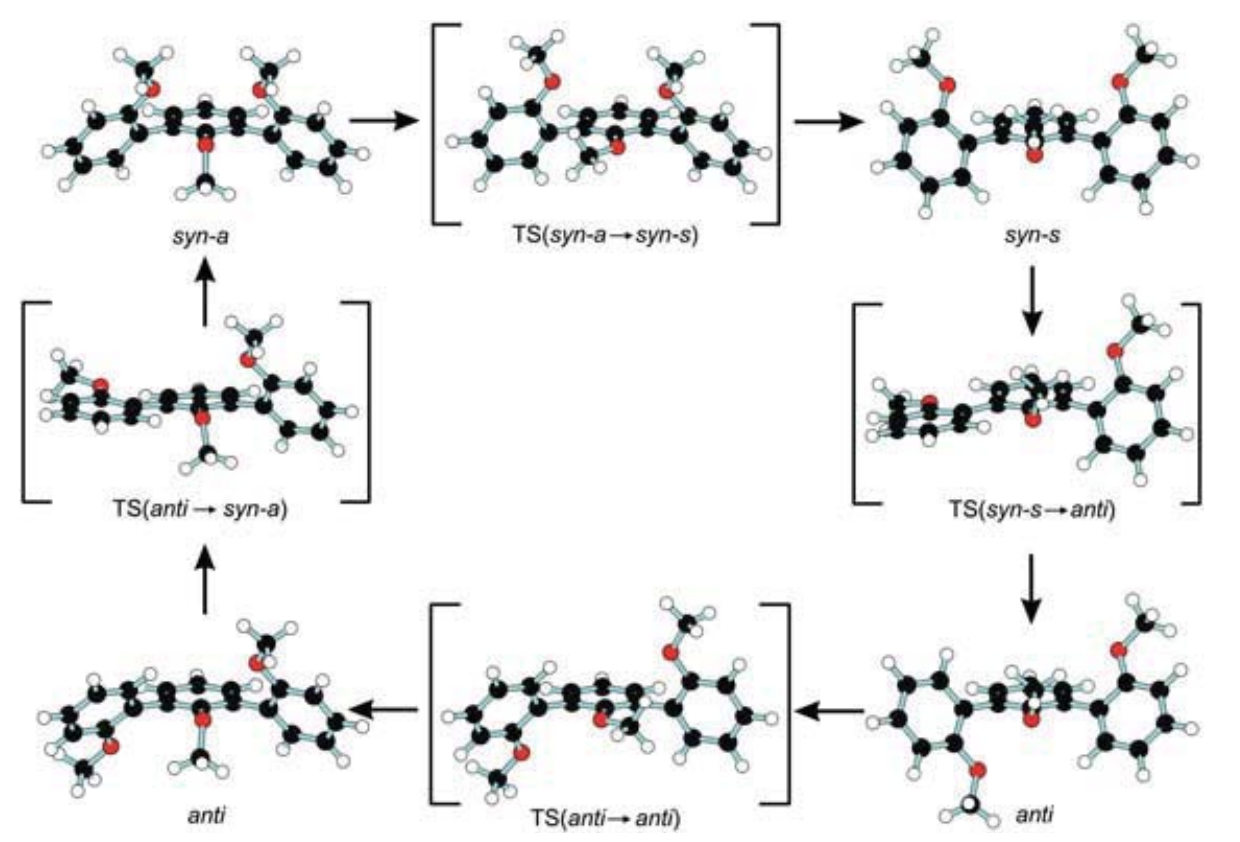

Figure 3. Interconversion of atropisomers of $\mathbf{1 d}$ (structures from B2PLYPD/cc-pVDZ calculations; note that the two anti structures are identical).

the central O-Me moiety, and the calculated $\Delta \mathrm{G}^{\neq}$are in excellent accord with the experimental free energy of activation of $41.2 \mathrm{~kJ} / \mathrm{mol}$. For $\mathbf{1} \mathbf{d}$ these barriers are lower than for $\mathbf{1} \mathbf{a}$ and $\mathbf{1} \mathbf{b}$ as it was expected according to interpretation given previously. ${ }^{[1]}$

Based on the geometries from B2PLYPD/cc-pVDZ calculations, the ${ }^{1} \mathrm{H}$ NMR chemical shifts for the O-Me moieties were calculated following the protocol outlined in ref 21 (see Table 2). The predicted chemical shifts for the lateral -OMe groups are within $0.1 \mathrm{ppm}$ of the experimental values, while for the central -OMe groups they are off by about $0.2 \mathrm{ppm}$.

If we assume that rotation around the central $\mathrm{Ph}-\mathrm{OMe}$ bond is rapid on the NMR timescale, i.e. the signals of the two syn conformers are averaged, even at $-84^{\circ} \mathrm{C}$, then the signals for the central OMe group should almost coincide ( $2.89 \mathrm{ppm}$ for the averaged syn-, and $2.91 \mathrm{ppm}$ for the anti conformer), which may explain why only one signal is seen for these protons. With

Table 1. Relative Energies in $\mathrm{kJ} / \mathrm{mol}^{\mathrm{a}}$ of Different Atropisomers of $\mathbf{1} \mathbf{d}$ and Barriers for Their Interconversion (See Figure 3 and Table S1 in the Supporting Information for Structures).

\begin{tabular}{|c|c|c|c|c|}
\hline \multirow[b]{2}{*}{ conformer } & \multicolumn{2}{|c|}{ gas phase } & \multicolumn{2}{|c|}{$\mathrm{SCRF} @ \mathrm{CH}_{2} \mathrm{Cl}_{2}$} \\
\hline & $H_{\text {rel }}$ & $G_{\text {rel }}$ & $H_{\text {rel }}$ & $G_{\text {rel }}$ \\
\hline syn-s & 1.13 & 0.44 & 1.39 & 0.60 \\
\hline syn-a & 0.96 & 1.16 & (0) & 0.10 \\
\hline anti & $(0)$ & (0) & 0.10 & (0) \\
\hline $\mathrm{TS}($ syn-a $\rightarrow$ syn-s) & 13.90 & 20.90 & 12.16 & 19.16 \\
\hline $\mathrm{TS}($ syn-s $\rightarrow$ anti) & 32.70 & 42.57 & 32.46 & 42.33 \\
\hline $\mathrm{TS}(\boldsymbol{a n t i} \rightarrow \boldsymbol{s y n}-\boldsymbol{a})$ & 33.82 & 43.88 & 33.61 & 43.67 \\
\hline $\mathrm{TS}($ anti $\rightarrow$ anti $)$ & 15.09 & 21.84 & 13.01 & 19.75 \\
\hline
\end{tabular}

Table 2. Calculated ${ }^{1} \mathrm{H}$ NMR Spectroscopic Chemical Shifts for O-Me groups in $\mathbf{1 d}^{\text {a }}$

\begin{tabular}{|lccc|}
\hline isomer & $\delta$ O-Me (central) $(\mathrm{ppm})$ & $\delta$ O-Me (1) (ppm) & $\delta$ O-Me (2) (ppm) \\
\hline syn-a & 2.85 & 3.90 & 3.90 \\
syn-s & 2.94 & 3.81 & 3.81 \\
anti & 2.91 & 3.81 & 3.85 \\
a ${ }^{2}$ using the GIAO/ WP04/cc-pVDZ method with $\mathrm{CH}_{2} \mathrm{Cl}_{2}$ as a continuum solvent, and the scaling factors given in ref 21.
\end{tabular}


regard to the OMe groups on the lateral phenyl rings, averaging is predicted to lead to a signal at $3.83 \mathrm{ppm}$ for the for the anti conformer and one at $3.86 \mathrm{ppm}$ for the two syn-conformers, under conditions where the syn- and anti-conformers do not interconvert rapidly on the NMR time scale.Although the absolute chemical shifts are off by ca. 0.08 ppm (which is well within expectations from these calculations), their difference is in good accord with the observed $\Delta \delta=0.026 \mathrm{ppm}$. The fact that the anticonformer is predicted to predominate in the equilibrium, may explain why at $-84^{\circ} \mathrm{C}$ the signal at lower field is more intense than the other one (see Fig. 2).

\section{METHODS}

\section{General}

All reactions were performed using standard Schlenk techniques under an atmosphere of argon. Dioxane was purified by distillation under $\mathrm{N}_{2}$ from potassium-benzophenone ketyl. Column chromatography was done using 32-63 $\mu$ flash silica gel following the method of Still et al. ${ }^{[11]}$ All $\mathrm{mp}$ are uncorrected. All ${ }^{1} \mathrm{H}$ variabletemperature and ${ }^{13} \mathrm{C}$ NMR spectra were obtained using an NMR spectrometer operating at a ${ }^{1} \mathrm{H}$ frequency of 299.956 $\mathrm{MHz}$, using a $5 \mathrm{~mm}$ Four-Nucleus probe. The ambient temperature without heating or cooling was $22-23{ }^{\circ} \mathrm{C}$. NMR chemical shifts and coupling patterns in the aromatic rings were elucidated by simulation and curve fitting using WinDNMR version 7.1.12 (Reich, H. J., University of Wisconsin, Madison, WI). Low-temperature experiments used dry nitrogen gas cooled to $77 \mathrm{~K}$ in a heat exchanger, and temperatures were calibrated using the ${ }^{1} \mathrm{H}$ shift separation of a methanol sample: $\mathrm{T}\left({ }^{\circ} \mathrm{K}\right)=409.0-$ $36.54 \mathrm{x}-21.85 \mathrm{x}^{2}$ where $\mathrm{x}$ is the chemical shift difference in ppm between $\mathrm{CH}_{3}$ and $\mathrm{OH}$ proton resonances. ${ }^{[23]}$ All ${ }^{1} \mathrm{H}$ NMR spectra are referenced to residual solvent at $\delta 5.32 \mathrm{ppm}\left(\mathrm{CHDCl}_{2}\right)$, and all ${ }^{13} \mathrm{C}$ NMR spectra are referenced to deuterated at $54.00\left(\mathrm{CD}_{2} \mathrm{Cl}_{2}\right)$. High resolution MS were obtained by direct insertion.

\section{Synthesis of 2,6-di(o-anisyl)anisole, 1d}

The method reported by Azzena and co-workers ${ }^{[12]}$ for Suzuki coupling was used with slight modification. To a solution of 1,3-dibromo-2-methoxybenzene ${ }^{[13,14]}$ (300 mg, $1.13 \mathrm{mmol}$ ) in 1,4-dioxane (1.5 mL) under $\mathrm{Ar}$, were added a $2 \mathrm{M}$ aqueous solution of $\mathrm{Na}_{2} \mathrm{CO}_{3}(1.13 \mathrm{~mL}, 2.26 \mathrm{mmol}), \mathrm{LiCl}(153 \mathrm{mg}, 3.61 \mathrm{mmol})$, 2-methoxyphenylboronic acid (686 $\mathrm{mg}, 4.5 \mathrm{mmol}$ ), and $\mathrm{Pd}$ $\left(\mathrm{PPh}_{3}\right)_{4}(130 \mathrm{mg}, 0.113 \mathrm{mmol})$. This mixture was stirred and heated at reflux for $24 \mathrm{~h}$. After cooling to room temperature, $\mathrm{H}_{2} \mathrm{O}(20 \mathrm{~mL})$ was added to the suspension. The aqueous layer was extracted with EtOAc ( $3 \times 20 \mathrm{~mL})$. The combined organic layers were washed successively with $1 \mathrm{M}$ aqueous $\mathrm{NaOH}$ $(50 \mathrm{~mL})$, brine $(50 \mathrm{~mL})$, and $\mathrm{H}_{2} \mathrm{O}(50 \mathrm{~mL})$. The organic layer was dried $\left(\mathrm{MgSO}_{4}\right)$ and then concentrated under reduced pressure. The residue was purified by silica gel chromatography using hexanes:chloroform (1:1) and then chloroform to elute $\mathbf{1 d}$ as a white solid (160 mg, 44\%). The product was further purified by recrystallization twice from diethyl ether:hexanes $(1: 1): \mathrm{mp}$ 118-119 ${ }^{\circ} \mathrm{C}$ (lit. $\left.{ }^{3} 117-118^{\circ} \mathrm{C}\right){ }^{1} \mathrm{H}$ NMR $\left(500 \mathrm{MHz}, \mathrm{CD}_{2} \mathrm{Cl}_{2}\right) \delta 3.143$ $(\mathrm{s}, 3 \mathrm{H}), 3.816(\mathrm{~s}, 6 \mathrm{H}), 7.016(\mathrm{dd}, J=8.3,1.2 \mathrm{~Hz}, 2 \mathrm{H}), 7.018$ $(\mathrm{dt}, J=1.1,7.6 \mathrm{~Hz}, 2 \mathrm{H}), 7.147(\mathrm{~A}, 1 \mathrm{H})$ and $7.231(\mathrm{~B}, 2 \mathrm{H}), \mathrm{AB}_{2}$ system $\left(J_{A B}=7.6 \mathrm{~Hz}\right), 7.293(\mathrm{dd}, J=7.7,1.8 \mathrm{~Hz}, 2 \mathrm{H}), 7.357$ (ddd, $J=8.2,7.5$, $1.8 \mathrm{~Hz}, 2 \mathrm{H}) .{ }^{13} \mathrm{C}$ NMR $\left(125 \mathrm{MHz}, \mathrm{CD}_{2} \mathrm{Cl}_{2}\right) \delta 56.00,60.75,111.34$,
120.79, 123.17, 128.61, 129.23, 131.66, 131.85, 132.54, 156.72 , 157.45; IR (KBr) 2934, 2833, 1581, 1491, 1465, 1272, 1237, 1025, $756 \mathrm{~cm}^{-1}$; HRMS (GC MS El ${ }^{+}, \mathrm{m} / \mathrm{z}$ ): Calcd for $\mathrm{C}_{21} \mathrm{H}_{20} \mathrm{O}_{3}, 320.1412$; Found: 320.1412 .

\section{Variable temperature NMR studies}

$\mathrm{CD}_{2} \mathrm{Cl}_{2}$ was used as the solvent. The ${ }^{1} \mathrm{H}$ NMR exchange line fitting results were obtained using WinDNMR version 7.1.14 (Reich, H.J., University of Wisconsin, Madison, WI). The spectra used for fitting were acquired just below the coalescence temperature for the resonance being fitted in order to maximize the information content. Line widths were determined by fitting peaks well below the coalescence temperature where exchange broadening is negligible. Chemical shifts and relative peak areas of the two conformers, as well as the exchange rate constants were obtained by visual fit to the experimental data. The conformer present in greater concentration just below coalescence is referred to as the " $\mathrm{A}$ " conformer, and the other conformer is described as " $B$ ". The free energy of activation was calculated using the equation: $\Delta \mathrm{G}^{\star}=[23.76-\ln (k / T)] R T^{[15]}$

\section{Quantum chemical calculations}

Geometry optimizations and frequency calculations were first done with the B3LYP/6-31G* method, where all stationary points (minima, transition states) were properly characterized and thermodynamic functions were calculated. Geometries were then reoptimized with the recently introduced "double hybrid" B2PLYP functional, ${ }^{[16]}$ corrected for contributions of dispersion energy, ${ }^{[17,18]}$ which accounts much better for steric interactions than pure DFT functionals do. As analytic second derivatives were not yet available for the B2PLYP method, we used the thermal corrections and entropies from B3LYP calculations in the evaluation of the relative enthalpies and free energies listed in Table 1. Finally, single-point SCRF calculations in $\mathrm{CH}_{2} \mathrm{Cl}_{2}$ as a continuum solvent were done, with the B2PLYP-D functional at the corresponding geometries, to evaluate relative enthalpies and free energies in solution. NMR chemical shifts were calculated using the GIAO method and the WPO4 functional that was developed with that purpose in mind, ${ }^{[19,20]}$ using the cc-pVDZ basis set. SCRF calculations were again carried out in $\mathrm{CH}_{2} \mathrm{Cl}_{2}$ as solvent and the raw isotropic magnetic shieldings (IMS) were converted to chemical shifts relative to TMS $\delta$ by scaling them with the parameters elaborated recently by Jain et al. ${ }^{[21]}(\delta=31.844$ - (IMS/1.0205)). All calculations were done with the Gaussian program package. ${ }^{[22]}$

\section{Acknowledgements}

We gratefully acknowledge financial support of this work by the U.S. National Science Foundation (CHE-0956581, R.S.G.) and the Swiss National Science Foundation (Project 2000-121747, T.B.).

\section{REFERENCES}

[1] U. I. Zakai, A. Błoch-Mechkour, N. E. Jacobsen, A. Abrell, G. Lin, G. S. Nichol, T. Bally, R. S. Glass, J. Org. Chem. 2010, 75, 8363-8371.

[2] H. O. House, J. A. Hrabie, D. VanDerveer, J. Org. Chem. 1986, 51, 921-929.

[3] L. Lunazzi, M. Mancinelli, A. Mazzanti, J. Org. Chem. 2007, 72, 5391-5394

[4] L. Lunazzi, M. Mancinelli, A. Mazzanti. J. Org. Chem. 2009, 74, 1345-1348.

[5] D. Casarini, L. Lunazzi, A. Mazzanti, Eur. J. Org. Chem. 2010, 2035-2056.

[6] D. J. Cram, I. B. Dicker, M. Lauer, C. B. Knobler, K. N. Trueblood, J. Am. Chem. Soc. 1984, 106, 7150-7167.

[7] G. Dyker, A. Kellner, J. Organomet. Chem. 1998, 555, 141-144. 
[8] N. Miyaura, A. Suzuki, Chem. Rev. 1995, 95, 2457-2483.

[9] H. J. Reich, WinDMR version 7.1.12, University of Wisconsin, Madison, WI.

[10] In one syn isomer the O-Me group of the central ring is facing away from the $\mathrm{MeO}$ groups and in the other the O-Me group is facing toward the MeO groups.

[11] W. C. Still, M. Kahn, A. Mitra, J. Org. Chem. 1978, 43, 2923-2925.

[12] J. Azzena, M. Cattari, G. Melloni, L. Pisano, Synthesis 2003, 2811-2814.

[13] F. F. Blicke, F. D. Smith, J. L. Powers, J. Am. Chem. Soc. 1932, 54, 1465-1471

[14] J. M. W. Chan, T. M. Swager, Tetrahedron Lett. 2008, 49, 4912-4914.

[15] H. Günther, NMR Spectroscopy, 2nd ed., Wiley, New York, 1992; 343.

[16] S. Grimme, J. Chem. Phys. 2006, 124, 034108/1-034108/16.

[17] T. Schwabe, S. Grimme, Phys. Chem. Chem. Phys. 2007, 9, 3397-3406.
[18] for an overview of these methods, see: T. Schwabe, S. Grimme, Acc. Chem. Res. 2008, 41, 569-579.

[19] K. W. Wiitala, T. R. Hoye, C. J. Cramer, J. Chem. Theory Comput. 2006, 2, 1092.

[20] In Gaussian, the WP04 functional is invoked by specifying the BLYP keyword and adding iop(3/76=1000001189,3/77=0961409999, $3 / 78=0000109999)$ to the keyword line.

[21] R. Jain, T. Bally, P. R. J. Rablen, Org. Chem. 2009, 74, 4017-4023.

[22] M. J. Frisch et al. Gaussian 03, Revision E.01, and Gaussian 09, Revision A.02, 2003, Gaussian, Inc., Wallingford, CT

[23] C. Ammann, P. Meier, A. E. Merbach, J. Magn. Reson. 1982. 46, 319-321. 